\title{
José Candell, Mikel San-Julian: Pediatric bone sarcomas
}

\author{
Springer-Verlag, Berlin, Heidelberg, New York, 2009, 161 pp, 86 figs., HardCover, \\ EUR 92, 95 CHF 144,50 USD 139,00 GBP 80,00, ISBN 978-1-84882-129-3
}

\author{
Claude Karger
}

Received: 20 December 2010/Accepted: 21 December 2010/Published online: 31 December 2010

(C) Springer-Verlag 2010

This manual addresses malignant bone tumors by focusing upon physeal distraction (epiphysiolysis) before excision. This technique has been used by the authors for a long time, with the goal to preserve the joint in metaphyseal malignant bone tumors in children. This original concept is now shared with the wider community of those in the field of treating malignant pediatric bone tumors.

For these patients, survival rates have increased, and the new challenges posed to the surgeon are on preserving the limb and its function, while avoiding complication.

The 11 chapters of the book cover all the required information to share the concepts developed by the authors: molecular biology, histological study of the barrier effect of the growth plate, imaging methods and histological findings, technical aspects, clinical results, and finally questions and answers.

Illustrations are abundant and clearly presented.

This book is targeted primarily toward orthopedic surgeons and pediatric oncologists, but it will also be of interest to all those in the fields of orthopedics, oncology, pediatrics, and surgery.

Conflict of interest No funds were received in support of this study. 